\title{
Injection devices for bulking agents in uro-gynaecology
}

\author{
Maria Angela Cerruto, Carolina D'Elia, Pierpaolo Curti \\ Urology Clinic, A.O.U.I. Verona, Italy.
}

\begin{abstract}
Summary Stress urinary incontinence (SUI) affects a large proportion of middle-aged and elderly women. When all conservative means are ineffective, a surgical treatment including retropubic suspension, pubovaginal and tension-free slings, is contemplated. Intra-urethral injections with bulking agents have been used as an alternative to the mentioned surgical procedures with alternate results. Many urethral bulking agents are available, such as bovine glutaraldehyde cross linked (GAX) collagen, polytetrafluoroethylene (Teflon), polydimethyl-sillxane elastomer (silicone), carbon coated zirconium beads, hyaluronic acid/dextranomer, and autologous tissues such as fat and cartilage.

These substances may be injected in a retrograde or antegrade fashion in the periurethral tissue and whether one route of injection is better than another is not well documented in the literature. We briefly describe the main injection techniques and devices of the most common bulking agents used in the treatment of female SUI.
\end{abstract}

KEY WORDS: Stress urinary incontinence; Bulking agents; Transurethral injections.

Submitted 3 October 2013; Accepted 31 december 2013

\begin{abstract}
INTRODUCTION
Stress urinary incontinence (SUI) affects a large proportion of middle-aged and elderly women, considerably lowering their quality of life and causing major economical costs to the society. When all conservative means are ineffective, a surgical treatment is contemplated, including retropubic suspension, pubovaginal and tension-free slings. Tensionfree tapes are considered minimally invasive procedures, yielding a lesser degree of discomfort and a faster return to normal daily activities for the patients, but they still require the use of the operating room, troncular anaesthesia, and an overnight hospital stay in most instances. Intra-urethral injections with bulking agents have been used as an alternative to the mentioned surgical procedures with alternate results, also due to the different characteristics of the various agents utilized, the most common shortcomings being lack of biocompatibility, allergenicity and short permanence in the tissue. Continuous advances in materials technology have provided the possibility that many urethral bulking agents are available (1), such as bovine glutaraldehyde cross linked (GAX) collagen, polytetrafluoroeth-
\end{abstract}

ylene (Teflon), polydimethyl-sillxane elastomer (silicone), carbon coated zirconium beads, hyaluronic acid/dextranomer, and autologous tissues such as fat and cartilage (2). These substances may be injected in a retrograde (more common) or antegrade fashion in the periurethral tissue around the bladder neck and proximal urethra.

Whether one route of injection is better than another is not well documented in the literature. Schulz et al. compared the transurethral injection route with paraurethral one (3). The Authors showed a trend towards better subjective and objective outcomes in favour of transurethral injection without reaching statistical significance.

Although there is no evidence that transurethral route is better than paraurethral, the argument in favour of the former is supported by the finding of more complications following paraurethral injection (4).

We briefly describe the main injection techniques and devices of the most common bulking agents used in the treatment of female SUI.

\section{TRANSURETHRAL AND PERIURETHRAL INJECTION TECHNIQUE AND DEVICES OF COLLAGEN}

The precise placement of the injected material is essential to ensure the most desired clinical outcome (5). The injection may be performed through a needle placed directly through the cystoscope or periurethrally with a spinal needle placed percutaneously at the introitus and positioned in the tissue adjacent to the urethra.

The collagen implant syringe contains $2.5 \mathrm{ml}$ of sterile bovine GAX collagen dispersed in phosphate buffered physiological saline. Transurethral injection is performed using a $18 \mathrm{Fr}$ cystoscope. The needle is advanced into the urethral wall just below the bladder neck and the implant is injected submucosally until the urethral coaptation is observed at the needle penetration site. Injections may be repeated at multiple sites (from 1 up to 5 injections) as needed until closure of the proximal urethral lumen is achieved (6).

Periurethral injection could minimize possible complications such as bleeding and extrusion of the injected substance (5). It should be easy to handle the spinal needle and cystoscope simultaneously to ensure precise placement of the needle tip in the periurethral tissue in the proximal urethra just below the bladder neck. The patient is placed in the lithotomy position, and after the anaesthetic procedures, a 20- or 22-gauge spinal needle, 
with the obturator in place, is placed at 4 o'clock position with the bevel of the needle directed medially toward the lumen of the urethra (5). The needle is advanced through the urethral muscle into the lamina propria, remaining entirely submucosally. In this plane, the needle should advance with ease. During needle advancement, a 17-18 Fr cystoscope employing either a 0 - or 30-degree lens may be used to ensure optimal needle placement. The needle should be positioned $0.5 \mathrm{~cm}$ below the vesical neck within the lamina propria (6). After removing the spinal needle obturator, the substance is injected with one hand while stabilizing the cystoscope with the other one. The collagen is seen accumulating first within the lining of the urethra as a whitish bulging of the urethral mucosa. The material should be injected slowly to allow accommodation within the tissues. When the urethra is $50 \%$ or greater occluded, the needle is removed and reinserted on the opposite side in the 8 o'clock position. Additional collagen may be injected until the urethral mucosa coapts, creating the desired urethral occlusion. To prevent remodelling of the injected material, care should be taken not to advance the cystoscope proximal to the injected material. Should the urinary bladder become over-distended during the injection process, the cystoscope may me removed and a 12Fr catheter inserted to drain the bladder (5).

\section{TRANSURETHRAL INJECTION TECHNIQUE AND DEVICES OF SILICONE MICROIMPLANTS (MACROPLASTIQUE; POLYDIMETHYLSILOXANE)}

Macroplastique is a soft tissue bulking agent and is comprised of soft, flexible, highly-textured irregularly shaped implants of heat vulcanized polydimethylsiloxane (a solid silicone elastomer) suspended in a bio-excretable carrier gel. The carrier gel is a pharmaceutical grade, water-soluble, low molecular weight polyvinylpyrrolidone (PVP or povidone) hydrogel which is absorbed by the reticuloendothelial system and excreted unchanged in the urine. Polydimethylsiloxane elastomer and polyvinylpyrrolidone have favorable biocompatibility properties. Polydimethylsiloxane is well tolerated by the cellular immune system and is non-genotoxic, non-carcinogenic and non-teratogenic (7).

The endoscopic injection procedure can be performed under general, regional or local anaesthesia. During the transurethral procedure, under direct cystoscopic vision, the needle, inserted through the cystoscope operative channel, is placed submucosally into the urethra at the six, ten and two o'clock positions 1.5 to $2.0 \mathrm{~cm}$ distal from the bladder neck. The material is slowly injected until a sufficient mucosal bleb is achieved. The procedure is completed if there is a total mucosal apposition together with occlusion of the urethral lumen. The injectable can also be administered periurethrally, under cystoscopic control by inserting a spinal needle percutaneously adjacent to the urethra. After injecting, the cystoscope should not be advanced past injected areas and only small 'in and out' catheters (8-12 Fr) should be inserted because this may result in compression or extrusion of the bulking agent afterwards. To improve and simplify the transurethral implantation technique and to minimize problems associated with endoscopic procedures, the Macroplastique Implantation System (MIS) was developed for the treatment of female SUI.

The device seems to allow a constant placement of the implants at predefined depth and angles at the six, ten and two o'clock position of mid-urethra within the same circumferential plane. To identify the site of implantation correctly, the ruler measuring scale on the topside of the device is used. The site of the bladder neck is identified as the position where the water flow from the fluid drainage channel ceases while the tip of the device is slowly withdrawn from the fluid-filled bladder. The standard implantation position is defined by withdrawing the device from the urethra to the appropriate location of the mid-urethra, i.e., $10-15 \mathrm{~mm}$ distance from the level of the bladder neck. Before withdrawing the needle, it is advisable to wait for at least 30 seconds in order to avoid any material leakage.

\section{TRANSURETHRAL INJECTION TECHNIQUE AND DEVICES OF ZIRCONIUM OXIDE BEADS (DURASPHERE)}

Durasphere injection is performed using pre-packaged syringes containing $1.0 \mathrm{~mL}$ Durasphere and a $18 \mathrm{G}$ needle delivery device. Urethral bulking agent injections are usually performed transurethrally at the level of the bladder neck under direct vision. More than one site may be injected. In 2003 Majar et al. described a modified technique for an easier implantation of this agent (8). After routine video monitored cystoscopy the bladder is completely drained. The cystoscope is withdrawn to the distal urethra, so that the mid-urethra, proximal urethra and bladder neck are viewed simultaneously. The needle is introduced through the cystoscopic sheath. With the bevel pointing toward the urethral lumen, the needle is directed at 45 degrees to the lumen and into the urethral wall at the 4 o'clock position (left hand dominant surgeons may find the 8 o'clock position preferable). After the needle tip penetrates the urethral wall and the bevel is no longer seen, the needle is advanced, this time parallel to the urethral lumen, for 1 to $2 \mathrm{~cm}$. At this point $1.5 \mathrm{ml} 1 \%$ lidocaine solution are injected into the submucosal layer. This step results in partial coaptation of the urethral walls and hydrodissection of the space to which the beads are eventually injected. Using this technique the whole circumference of the urethra coapts with no need to change the needle location from its original 4 o'clock position. The bulking agent is then injected with steady and consistent thumb or thenar eminence pressure on the plunger using 1 hand, while holding the cystoscope with the contralateral hand.

Resistance to injection at this point is managed by gradual withdrawal/advancement of the needle tip and by turning the bevel in a clockwise or counterclockwise direction. Insistence on injecting the beads at exactly the same location and bevel orientation would result in further bead impaction and limits the capability of bead delivery. Complete needle withdrawal is avoided. This step is repeated as needed with the surgeon steadily holding the delivery system and maintaining the needle at exactly the same location under cystoscopic guidance: The assistant replaces the used syringe with a new, full syringe, so that further material can be injected (usually 
2 to 3 syringes are needed) until nearly complete urethral lumen coaptation is achieved. When almost complete coaptation is attained, the syringe is replaced and $1.5 \mathrm{ml}$ normal saline are injected at the same position. This step enables beads remaining in the needle to be deployed at the injection site. The needle tip is then maintained at the same position for another 10 seconds. At the end of the procedure the bladder is emptied using a 12Fr straight catheter. Carbon bead bulking agent may be injected also periurethrally (9). A rigid $0^{\circ}$ cystoscope is introduced into the urethra to allow visualization of the bladder neck. A 1.5-in, bent, $18 \mathrm{G}$ needle is then inserted periurethrally at either 4-o'clock or 8-o'clock position at approximately $0.5 \mathrm{~cm}$ distal to the bladder neck.. Then the material is injected until either the luminal appearance demonstrates mucosa coaptation or $6 \mathrm{~mL}$ of the product has been used. If good circumferential coaptation of the tissue has been achieved by injecting the carbon beads from just one injection site, the needle is not inserted on the other side.

\section{TRANSURETHRAL INJECTION TECHNIQUE AND DEVICES OF NASHA/DX COPOLYMER (ZUIDEX)}

NASHA/Dx copolymer comprises dextranomer (Dx) microspheres (80 to $250 \mathrm{~m}$ ) in a carrier gel of non-animalstabilized hyaluronic acid (NASHA). The gel is a biocompatible, biodegradable material free of animal products, has no immunogenic properties, and has been shown not to migrate to different organs after submucosal injection. A guiding instrument, named the Implacer, has been developed to facilitate reproducible and standardized transurethral injection of NASHA/Dx copolymer, without the need for surgical facilities or cystoscopic guidance (10). Zuidex ${ }^{\mathrm{TM}}$ gel is a Dextranomer/hyaluronic acid (Dx/HA) copolymer. Dextranomer (Dextran 2.3 dihydropropyl 2-hydroxy-1.3 propane-diethylethers) is made by hydrophylic dextran polymer particles (microspheres 80-120 micron), configurated as a network. It acts as a cell carriers, recruiting connective fibers from the surrounding tissues. It is non-alergic, as it has no free dextran molecules. Hyaluronic acid is a $1 \%$ solution, highly viscous, highly molecular weight polysaccaride. It is non-immugenic as it is not extracted from animals but from bacteria. The Zuidex System is composed of: 4 pre-filled syringes, each containining $0.7 \mathrm{~mL}$ of Zuidex ${ }^{\mathrm{TM}}$ gel, and a Zuidex Implacer. The latter is a plastic device that consists of i) one hand piece, ii) a head with 4 thin channels where the $21 \mathrm{G}$ needles of the 4 syringes are passed, and iii) a specially designed sliding cannula. The cannula covers the needles for the smooth insertion into the urethral lumen and once withdrawn, enables the prick of the urethral submucosa in 4 sites. The procedure may not require the use of the operating room and it is made on an outpatient basis, in the office, under local anaesthesia. Nevertheless, general and/or local anaesthesia may be administered, depending on investigator preference.

The first step is the urethral length measurement. This is done by means of a Foley catheter with the filled balloon gently snuggled against the bladder neck, by marking the catheter surface at the exit from the external meatus. In an office setting, only $5 \mathrm{mg}$ of anaesthetic gel are instilled into the urethra and left in place for 10 minutes. The Implacer and the four syringes are assembled. The needles are thereafter covered by the cannula pushed to its bottom position. At this point the distal tip of the cannula is advanced through the meatus as far as the mid-urethra, and is firmly kept in this position throughout the whole procedure. The cannula is slid backward to uncover the needles in the urethral lumen. Each needle is withdrawn 1 $\mathrm{cm}$ and push forward again to perforate the urethral mucosa and the Zuidex gel is injected into the urethral wall. This manoeuvre may be started at 2 o'clock position in a clockwise direction. Subsequently, all the syringes and the Implacer itself are removed. No transurethral catheter is used after the procedure. The patient is observed until she voids with no significant residual urine.

\section{Conclusions}

As bulking materials develop, understanding of the preferred injection technique also is being gained, as well as the best delivery method and injection site. Every effort should be done for making these devices safe and easy to manage, more and more.

\section{REFERENCES}

1. Appell RA, Dmochowski RR, Herschorn S. Urethral injections for female stress incontinence. BJU Int. 2006; (98 Suppl) 1:27-30.

2. Smith ARB, Daneshgari F, Dmochowski R, et al. Surgery for urinary incontinence in women. In: Abrams P, Cardozo L, Khoury S, Wein A: Incontinence. 3rd edition 2005; 1297-1370.

3. Schulz JA, Nager CW, Stanton SL, Baessler K. Bulking agents for stress urinary incontinence: short-term results and complications in a randomized comparison of periurethral and transurethral injections. Int Urogynecol J Pelvic Floor Dysfunct. 2004; 15:261-5.

4. Pickard R, Reaper J, Wyness L, et al. Periurethral injection therapy for urinary incontinence in women. Cochrane Database of Systematic Reviews 2003, Issue 2. CD003881.

5. Winters JC, Appell R. Periurethral injection of collagen in the treatment of intrinsic sphincter deficiency in the female patient. Urol Clin North Am. 1995; 22:673-678.

6. Groutz A, Blaivas JG, Kesler SS, et al. Outcome results of transurethral collagen injection for female stress incontinence: assessment by urinary incontinenc score.J Urol. 2000; 164:2006-9.

7. ter Meulen PH, Berghmans LCM, van Kerrebroeck P. Systematic review: efficacy of silicone microimplants (Macroplastique $\left.{ }^{\circledR}\right)$ Therapy for stress urinary incontinence. Eur Urol. 2003; 44:573-582.

8. Madjar S, Covington-Nichols C, Secrest CL. New periurethral bulking agent for stress urinary incontinence: modified technique and early results. J Urol. 2003; 170: 2327-2329.

9. Kershen RT, Dmochowski RR, Appell RA. Beyond collagen: injectable therapies for the treatment of female stress urinary incontinence in the new millennium. Urol Clin North Am. 2002; 29:559-574.

10. von Kerrebroeck P, ter Meulen P, Larsson G, et al. Efficacy and safety of a novel system (NASHA/Dx copolymer using the implacer device) for treatment of stress urinary incontinence. Urology. 2001; 58:12-15.

\section{Correspondence}

Maria Angela Cerruto,MD - mariaangela.cerruto@univr.it

Carolina D’Elia, MD,F.E.B.U. (Corresponding Author)-karolinedelia@gmail.com Pierpaolo Curti, MD - curtipierpaolo@gmail.com

Urology Clinic, A.O.U.I. Verona, Policlinico G.B. Rossi

Piazzale L.A. Scuro 10 - 37134 Verona, Italy 